\title{
Endovascular Treatment of Intracranial Vertebral Artery Dissecting Aneurysms: Follow up Angiographic and Clinical Results of Endovascular Treatment in Serial Cases
}

\author{
Gi Won Shin, MD, Hae Woong Jeong, MD
}

Purpose: To report angiographic and clinical results of endovascular treatment in 45 intracranial vertebral artery dissecting aneurysms (VADAs).

Materials and Methods: From July 2002 to September 2013, a total of 42 patients with 45 VADAs received endovascular treatment. Endovascular treatment consisted of internal trapping with detachable coils, stent-assisted coiling, and stenting only. Immediate and follow-up angiographic findings and clinical outcome were retrospectively reviewed.

Results: There were 17 ruptured VADAs and 28 unruptured VADAs. Overall, 26 VADAs were treated with internal trapping, 14 with stent-assisted coil embolization, and 5 with stenting only. Immediate angiographic results revealed complete occlusion in 31 cases and incomplete occlusion in 14 cases. Follow-up imaging studies were available in 31 cases. On follow-up imaging, antegrade recanalization occurred in 2 of 16 cases treated with internal trapping. Aneurysmal recurrence occurred in one case treated with stent-assisted coiling. Procedural complications occurred in 8 patients. In cases of unruptured VADA, favorable outcome (mRS 0 and 1 ) was achieved in 26 cases and poor outcome (mRS 2-5) in 2 cases. There was no mortality in patients with unruptured VADAs.

Conclusion: Endovascular treatment of intracranial VADA appears to be safe and effective. Follow-up angiographic study is needed because parent artery recanalization or aneurysmal recurrence can occur.

Key Words : Vertebral artery; Dissecting aneurysm; Endovascular treatment; Coil embolization

All authors: Department of Diagnostic Radiology, Busan Paik Hospital, Inje University, Busan, Korea

Received November 28, 2014; accepted after revision January 14, 2015.

Correspondence to: Hae Woong Jeong, MD, Department of Diagnostic Radiology, Inje University, Busan Paik Hospital, $633-165$ Kegumdong, Busanjin-ku, Busan 614-735, Korea.

Tel. 82.51.890.6549 Fax. 82.51.896.1085

E-mail: hwjeong2000@lycos.co.kr

This is an Open Access article distributed under the terms of the Creative Commons Attribution Non-Commercial License (http://creativecommons.org/licenses/by-nc/3.0) which permits unrestricted non-commercial use, distribution, and reproduction in any medium, provided the original work is properly cited. 


\section{Endovascular Treatment of Intracranial Vertebral Artery Dissecting Aneurysms}

Ruptured vertebral artery dissecting aneurysms (VADAs) presented with acute subarachnoid hemorrhage (SAH) have a high incidence of rebleeding and mortality rate compared with saccular aneurysms $[1,2]$. Immediate treatment with surgical or endovascular approach is necessary to prevent devastating rebleeding [3]. Surgical clipping of VADAs has several limitations because of deep location of aneurysms and their close relationship to lower brain stem and lower cranial nerves [4].

Unruptured VADAs may present with severe occipital headache or focal neurologic deficits due to vertebrobasilar ischemia without aggressive clinical course. The optimal treatment of unruptured VADA is still unknown $[3,5]$. As the reported risk of bleeding from unruptured VADA in recent studies is higher than those in the previous reports, endovascular treatment should be considered in cases which have unfavorable angiographic features $[1,6]$.

Follow-up imaging findings of untreated VADAs, such as gradual disappearance of the luminal narrowing or progressive enlargement of the aneurysmal dilatation, have been reported $[7,8]$. However, the results of serial angiographic follow-up combined with clinical outcomes after endovascular treatment especially with stent-assisted coiling have been rarely reported. Thus, the purpose of this study was to report immediate and follow-up angiographic results and clinical outcomes in 45 VADAs, which were managed with various endovascular techniques.

\section{MATERIALS AND METHODS}

\section{Patients}

Institutional Review Board approval was obtained, with informed consent being waived. From July 2002 to September 2013, a total of 42 patients having 45 VADAs were treated with endovascular treatment at single institution. The study group consisted of 23 males and 19 females (mean age, 52 years; age range, 36 to 80 years). Of the 45 VADAs, 17 were ruptured aneurysms and 28 were unruptured.

\section{Endovascular Treatment}

Decision of endovascular treatment method was based on the angiographic characteristics of VADAs, presence or absence of $\mathrm{SAH}$, and clinical presentations. Internal trapping by detachable coils was primarily considered when the VADA was located in proximal or distal to origin site of PICA. Stent implantation or

Table. Results of Immediate and Follow up Angiography after Endovascular Treatment in Vertebral Artery Dissecting Aneurysm

\begin{tabular}{|c|c|c|c|c|c|}
\hline \multicolumn{6}{|c|}{ Ruptured VADA $(n=17)$} \\
\hline Treatment method & $\begin{array}{l}\text { Immediate angiographic } \\
\text { result }\end{array}$ & & $\begin{array}{c}\text { Follow up } \\
\text { images }(n=9)\end{array}$ & $\begin{array}{c}\text { Follow up angiographic } \\
\text { result }\end{array}$ & \\
\hline \multirow{2}{*}{$\begin{array}{l}\text { Internal trapping } \\
(n=13)\end{array}$} & Complete occlusion & 13 & \multirow[t]{2}{*}{7} & Complete occlusion & 6 \\
\hline & Incomplete occlusion & 0 & & Antegrade recanalization & 1 \\
\hline \multirow{2}{*}{$\begin{array}{l}\text { Stent-assisted coil } \\
\text { embolization }(n=4)\end{array}$} & Complete occlusion & 1 & \multirow[t]{2}{*}{2} & No change & 1 \\
\hline & Incomplete occlusion & 3 & & Complete with vessel remodeling & 1 \\
\hline \multicolumn{6}{|c|}{ Unruptured VADA $(\mathrm{n}=28)$} \\
\hline Treatment method & $\begin{array}{c}\text { Immediate angiographic } \\
\text { result }\end{array}$ & & $\begin{array}{c}\text { Follow up } \\
\text { images }(n=22)\end{array}$ & $\begin{array}{c}\text { Follow up angiographic } \\
\text { result }\end{array}$ & \\
\hline \multirow{2}{*}{$\begin{array}{l}\text { Internal trapping } \\
(n=13)\end{array}$} & Complete occlusion & 13 & \multirow[t]{2}{*}{9} & Complete occlusion & 8 \\
\hline & Incomplete occlusion & 0 & & Antegrade recanalization & 1 \\
\hline \multirow{3}{*}{$\begin{array}{l}\text { Stent-assisted coil } \\
\text { embolization }(n=10)\end{array}$} & Complete occlusion & 4 & \multirow[t]{3}{*}{9} & Complete occlusion with vessel remodeling & 3 \\
\hline & Incomplete occlusion & 6 & & No change & 5 \\
\hline & & & & Recurrence & $1^{*}$ \\
\hline \multirow[t]{3}{*}{ Stent-only $(n=5)$} & \multirow[t]{3}{*}{ NA } & 5 & \multirow[t]{3}{*}{4} & Complete occlusion with vessel remodeling & 2 \\
\hline & & & & Decrease of size & 1 \\
\hline & & & & No change & 1 \\
\hline
\end{tabular}

${ }^{*}$ Retreatment with internal trapping 


\section{Gi Won Shin, et al.}

stent-assisted coil embolization was considered in cases of unruptured aneurysms with posteroinferior cerebellar artery (PICA) involvement. As a result, various endovascular strategies were used: internal trapping with coils $(n=26)$, stent-assisted coil embolization $(\mathrm{n}=14)$, and stent implantation only $(\mathrm{n}=5)$.

\section{Imaging Analysis}

MRI and angiographic findings were evaluated by single interventional neuroradiologist. The diagnosis of VADA was based on the characteristic appearances revealed on MRA, computed tomography angiography (CTA) or conventional angiography. VADA was located in right vertebral artery in 28 cases and in left vertebral artery in 17 cases. Involved vertebral artery was dominant artery in 10 cases and non-dominant in 19 cases. No vertebral artery dominance in 16 cases. 19
VADAs were located distal to the origin of PICA, 18 VADAs were proximal to that of PICA, and 8 VADAs were PICA involvement type. Immediate angiographic results were classified as complete or incomplete occlusion based on the completion angiography. Follow-up angiographic results were evaluated with CTA, MRA, or DSA more than 3 months later. These results were classified as complete occlusion, antegrade recanalization, no change, complete occlusion with vessel remodeling, and recanalization.

\section{Analysis of the clinical results}

The clinical data was reviewed retrospectively by a neurologist using the Hunt and Hess grading system (H\&H grade) and modified Rankin Scale (mRS) at 3months. Clinical outcomes were assessed at 3 months after endovascular treatment. In patients with
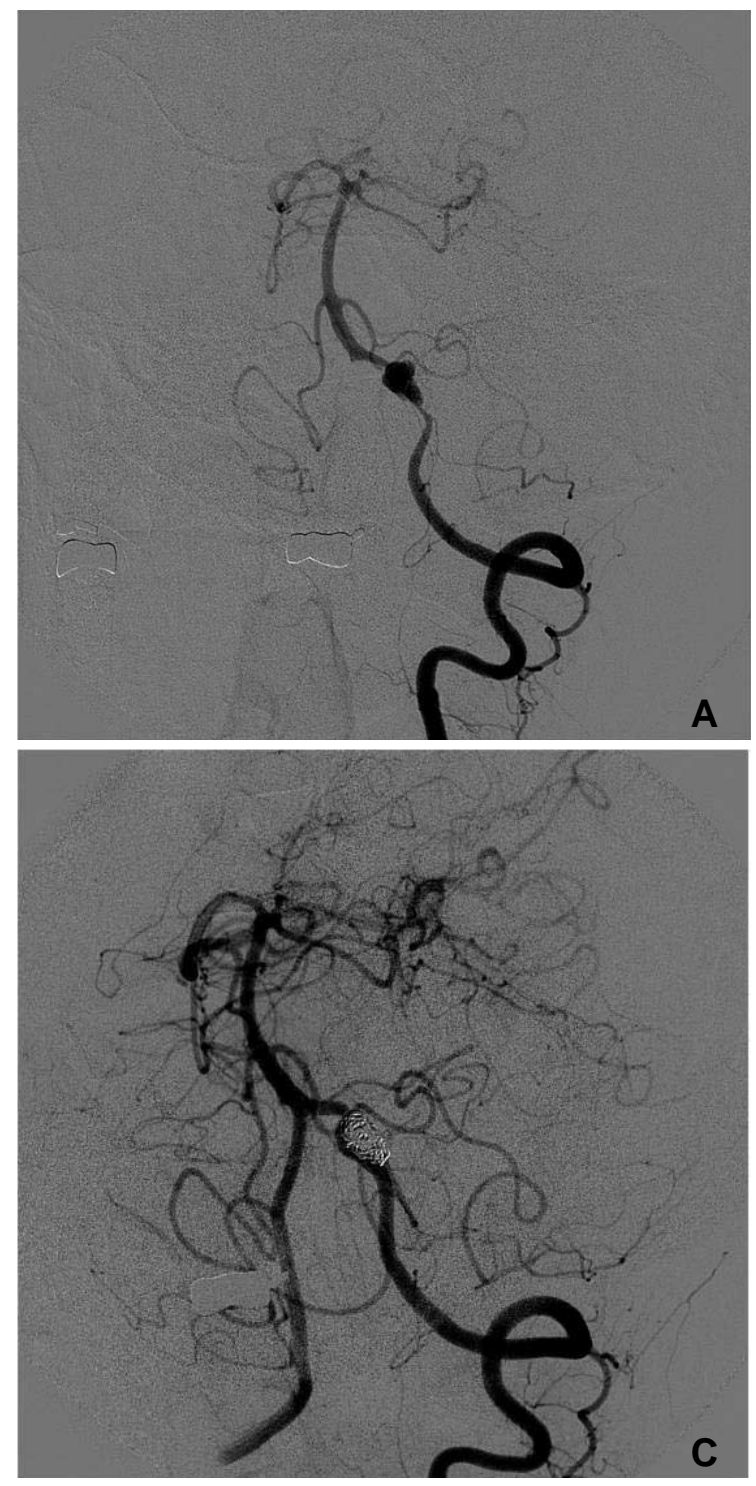

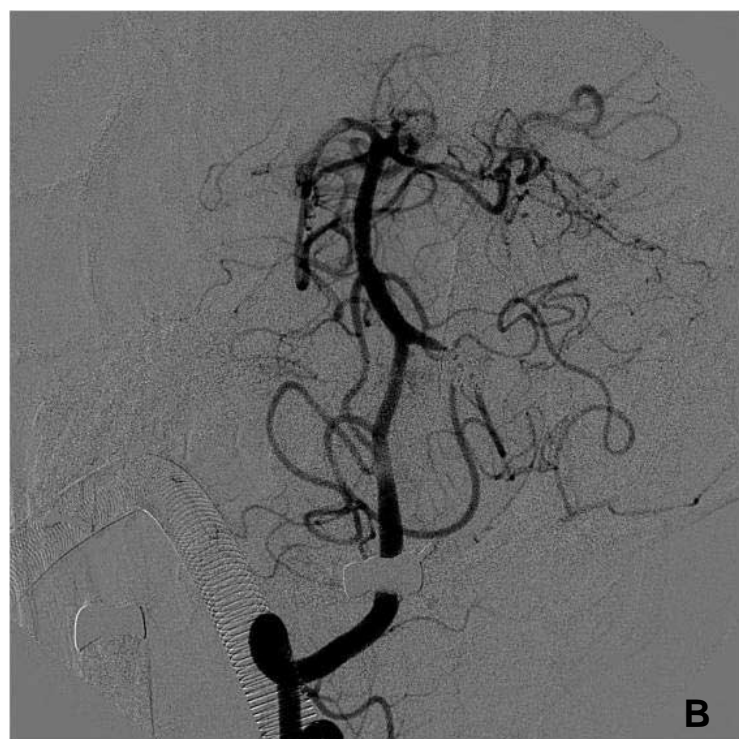

Fig. 1. A 41-year-old female with acute SAH. A. Working projection of the left vertebral angiogram shows a dissecting aneurysm arising from the left vertebral artery proximal to origin of PICA. B. The dissecting aneurysm and affected left vertebral artery are completely occluded. C. Follow-up angiogram after 6 months reveals recanalization of the occluded left vertebral artery with a normal arterial configuration and antegrade flow into the basilar artery. 


\section{Endovascular Treatment of Intracranial Vertebral Artery Dissecting Aneurysms}

unruptured VADA, favorable outcome was defined as mRS 0 and 1 .

\section{RESULTS}

\section{Ruptured vertebral artery dissecting aneurysm}

Of 17 ruptured VADAs, 5 cases initially presented with poor grades (4 or 5), 8 cases with grade 3, 2 cases with grade 2 , and 1 patient with grade 1 . Results of immediate and follow up imaging studies in ruptured VADAs are presented in the table. In ruptured cases $(n=17)$, endovascular treatment consisted of internal trapping with coil embolization $(\mathrm{n}=13)$ and stentassisted coil embolization using self-expandable stent $(n=4)$. Immediate angiographic results revealed complete occlusion in 14 cases and incomplete occlusion in 3 cases. Eight cases were lost to follow up.
Follow up imaging study in 7 cases treated with internal trapping revealed complete occlusion in 6 cases and antegrade recanalization in 1 case (Fig. 1). In 2 cases treated with stent-assisted coil embolization, follow up imaging studies showed no change in 1 case and complete occlusion with vessel remodeling in 1 case (Fig. 2). There were 6 procedure-related complications: lateral medullary infarction $(n=2)$, embolic infarction $(\mathrm{n}=2)$, unintended arterial occlusion $(\mathrm{n}=1)$, and parent artery dissection $(\mathrm{n}=1)$. Follow-up mRS was available in 13 patients. Favorable outcome (mRS 0 and 1) was achieved in 5 patients and poor outcome in 8 patients. Four patients died.

\section{Unruptured vertebral artery dissecting aneurysm}

Of 28 unruptured VADAs, 25 VADAs initially presented with headache, 2 with hemiparesis, and 1
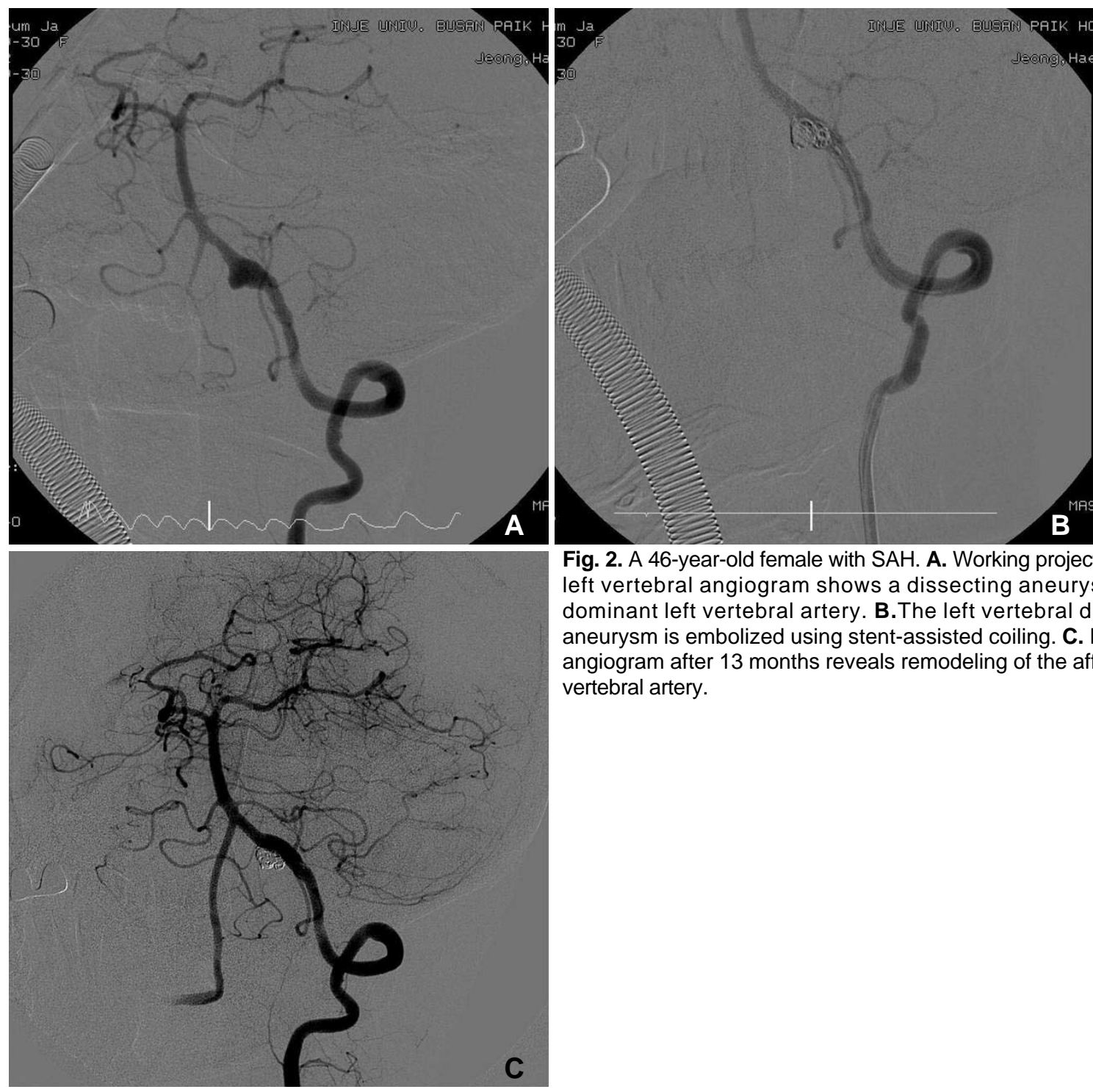

Fig. 2. A 46-year-old female with $\mathrm{SAH}$. A. Working projection of the left vertebral angiogram shows a dissecting aneurysm in the dominant left vertebral artery. B.The left vertebral dissecting aneurysm is embolized using stent-assisted coiling. C. Follow-up angiogram after 13 months reveals remodeling of the affected left vertebral artery. 
Gi Won Shin, et al.
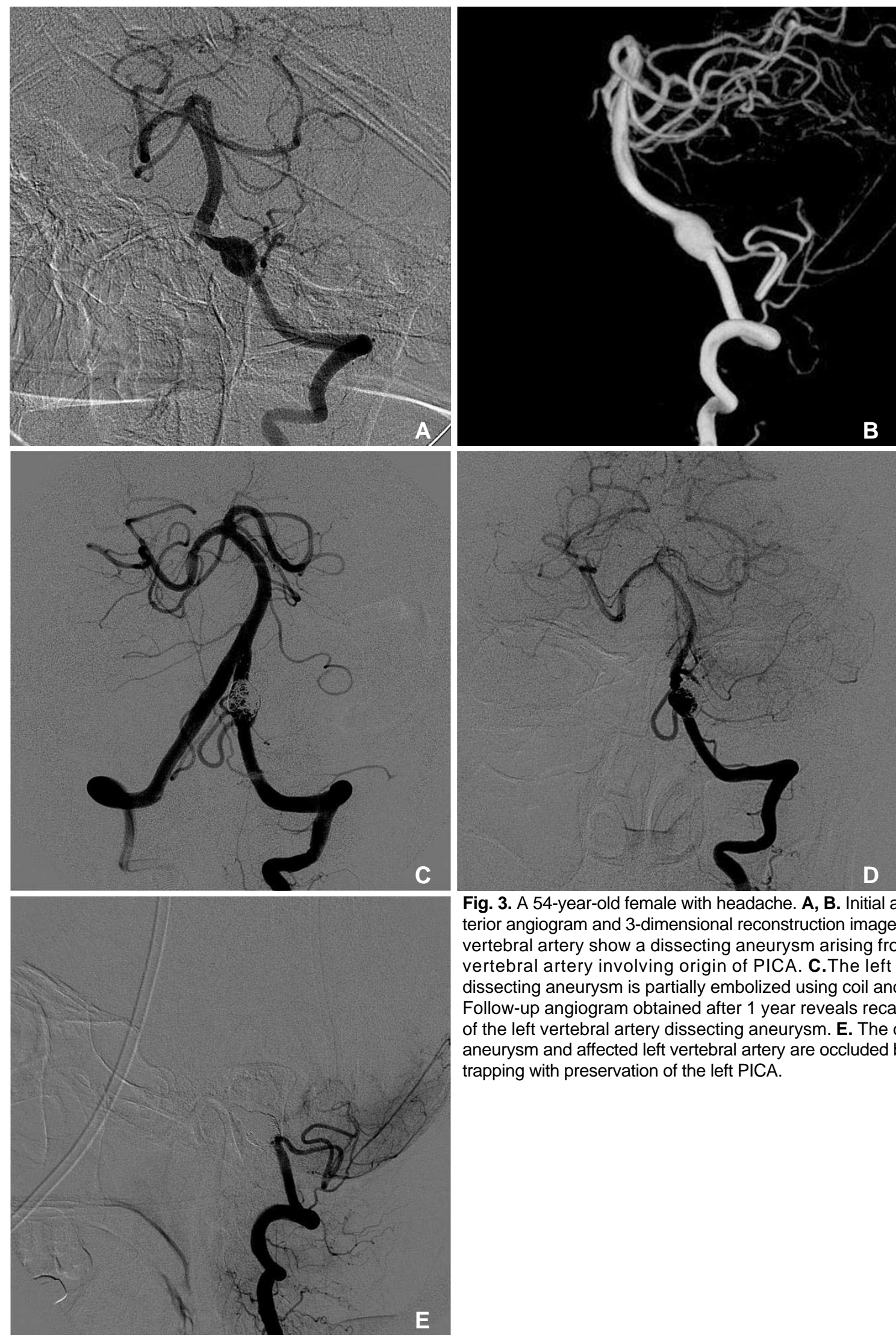

Fig. 3. A 54-year-old female with headache. A, B. Initial anteroposterior angiogram and 3-dimensional reconstruction image of the left vertebral artery show a dissecting aneurysm arising from the left vertebral artery involving origin of PICA. C.The left vertebral dissecting aneurysm is partially embolized using coil and stent. $\mathbf{D}$. Follow-up angiogram obtained after 1 year reveals recanalization of the left vertebral artery dissecting aneurysm. $\mathbf{E}$. The dissecting aneurysm and affected left vertebral artery are occluded by internal trapping with preservation of the left PICA. 


\section{Endovascular Treatment of Intracranial Vertebral Artery Dissecting Aneurysms}

with lateral medullary infarction. Results of immediate and follow-up imaging studies in unruptured VADAs are presented in table. In unruptured cases, internal trapping was performed in 13 cases, stent-assisted coil embolization in 10 cases, and stent implantation only in 5 cases. All VADAs treated with internal trapping showed complete occlusion on immediate postprocedural angiography. Immediate angiographic results in 10 cases treated with stent-assisted coliling showed complete occlusion in 4 cases and incomplete occlusion in 6 cases. Stent implantation was successful in all 5 cases treated with stenting only.

Follow-up angiographic studies were available in 22 cases. In 9 cases treated with internal trapping, complete occlusion occurred in 8 cases and antegrade recanalization in 1 case. In 9 cases treated with stentassisted coil embolization, complete occlusion with vessel remodeling occurred in 3 cases, no change in 5 cases, and recanalization in 1 case. Of these, a patient with recanalization on follow-up after stent-assisted coil embolization underwent retreatment with internal trapping using detachable coils (Fig.3). In cases treated with stenting only, complete occlusion with vessel remodeling occurred in 2 cases (Fig. 4), decrease of sac in size in 1 case, and no change in 1 case.

Follow-up mRS was available in all patients. Favorable outcome (mRS 0-1) was achieved in 26 cases and poor outcome (mRS 2-5) in 2 cases. There
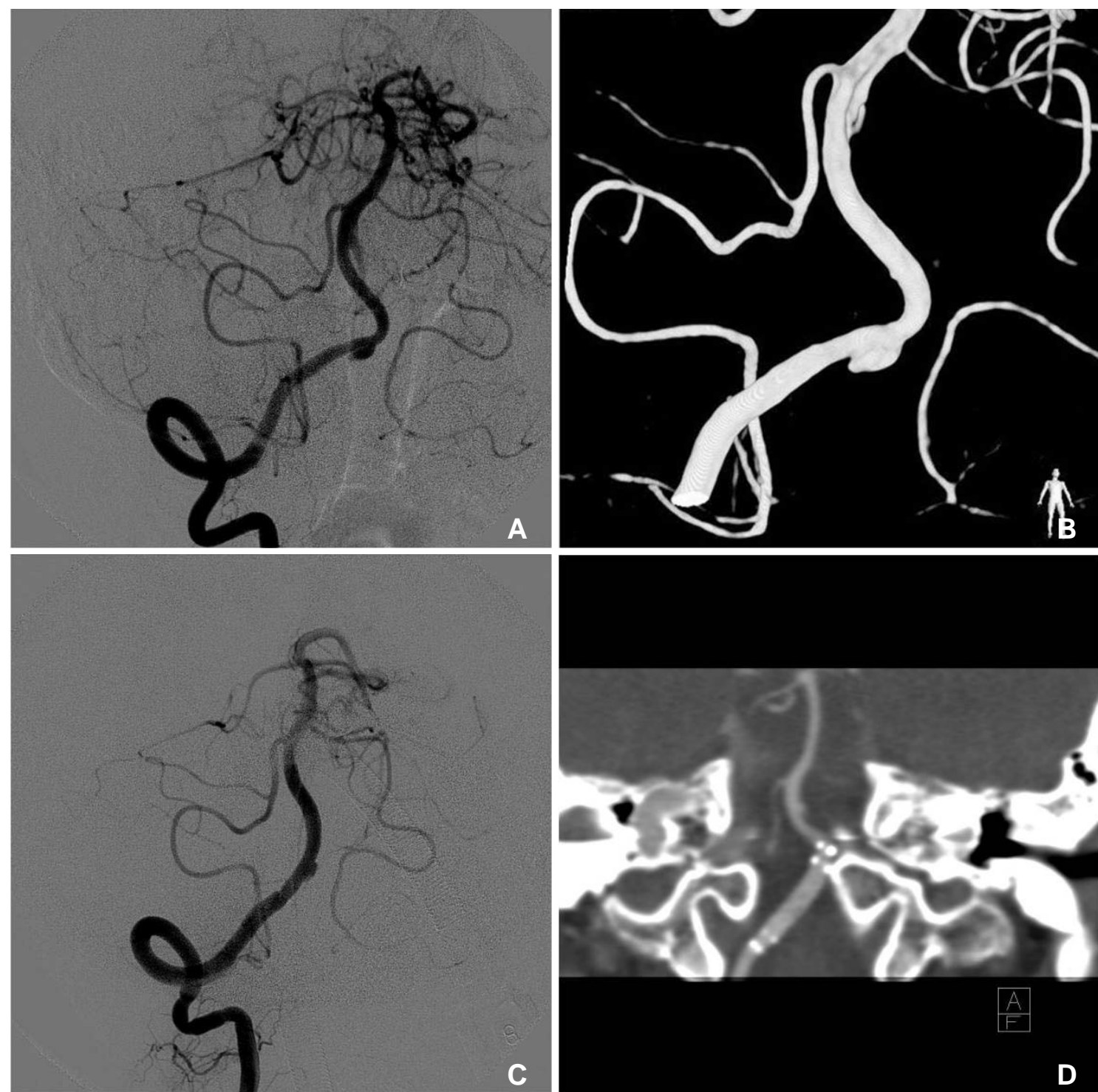

Fig. 4. A 41-year-old male with headache. A, B. Initial right vertebral angiogram and 3-dimensional reconstruction image of the right VA shows a dissecting aneurysm arising from the right VA. C. The double stent insertion without coil embolization in the affected right vertebral artery was done. D. Follow-up CT angiogram obtained after 11 months shows patent and improved blood flow with normalized vessel remodeling in stent inserted parent artery and its distal portion. 


\section{Gi Won Shin, et al.}

was no mortality. There was one case of lateral medullary infarction as a post-procedural complication.

\section{DISCUSSION}

Primary mechanism of the arterial dissection is disruption of the internal elastic lamina. After disruption, leukocyte and macrophage infiltration, endothelial coverage, and neointima formation follow subsequently [3]. SAH occurs due to rupture of the thin layer of intradural adventitia [9]. Ruptured VADA shows high incidence (over $70 \%$ ) of rebleeding within 24 hours which results in poor clinical outcome $[3,9,10]$. Endovascular intervention is mandatory to prevent these fatal rebleeding. Two main concepts in the endovascular treatment of the VADA are deconstructive and reconstructive techniques for the parent vessels. First, deconstructive techniques can be performed by balloon or coil embolization [11-16]. Multiple studies regarding direct occlusion of the pathologic segment were reported [17, 18]. However, there were several case reports of antegrade recanalization after endovascular treatment for ruptured VADAs $[19,20]$. The mechanism of the antegrade recanalization can be explained as following [10]; (1) if microcatheter navigate into the false lumen and occlude the false lumen with detachable coils, true lumen dilates into the normal diameter as false lumen collapses (2). Detachable coils were insufficient to cover the entry of the dissecting aneurysm. In our case, there was one ruptured VADA case which showed antegrade recanalization 6 months after internal trapping (Fig. 1).

Especially in case of VADA involving PICA in dominant side, alternative technique such as proximal occlusion can be considered. However, proximal occlusion technique can cause retrograde flow from the contralateral vertebral artery resulting in rebleeding [14]. In our study, although there were 8 cases of VADAs involving PICAs, none of those was treated in proximal occlusion technique.

Reconstructive techniques were applied to VADAs with stenting or stent-assisted coiling. Canton et al. [21] and Lopes et al. [22] reported the mechanism of endovascular stent deployment in VADA. They suggested flexible stents reduces the stresses acting on the aneurysm wall which results in true lumen expansion and parent artery reconstruction finally. Histologically, complete endothelialization of the stent and fibroelastic tissue formation along the aneurysmal neck explain mechanism of the stent deployment strategy. In recent reports, outcome of endovascular treatment with stenting in ruptured or unruptured VADA is favorable [1].

Although unruptured VADAs usually show benign clinical course, the natural course of the unruptured VADAs is still unknown and treatment guideline remains controversial. Administration of antiplatelet or anticoagulant in patients with unruptured VADAs and ischemic presentations may exacerbate aneurysmal dissection and cause rupture of the aneurysm. Risk of bleeding from unruptured VADA was higher than previous reports and endovascular treatment should be considered in some cases, such as in cases of increased size of aneurysmal dilatation during follow-up period and when double lumen sign is visible in the acute stage[6]. For making a decision of parent artery occlusion in cases of unruptured VADA, there are several factors to be considered: risk of bleeding in conservative treatment; risk of ischemic complications during procedure; blood supply from the anterior and posterior spinal artery, perforating arteries, and patency of contralateral vertebral artery; and relationship between aneurysm and PICA [3]. Reconstructive techniques, stent placement with or without coil embolization, aims at the preservation of the vertebral artery flow. This technique, as well as parent artery occlusion, show favorable results in unruptured VADA cases according to several reports [4, 23]. In our study, clinical and radiologic results were satisfactory in upruptured patients treated with internal trapping and stent placement with or without coils. Only one case revealed recanalization of the dissecting aneurysm after 1 year, which was retreated with internal trapping (Fig. $3)$. In one case, there was lateral medullary infarction 2 days after internal trapping. Although PICA has rich pial anastomoses, there have been case reports of PICA infarction on follow up images after endovascular procedure [3]. They suggested that thromboembolism due to coil loop herniation into the PICA origin and occlusion of a perforating artery was possible causes of the PICA territory infarction. However, as well as procedural complication, recurrent ischemia in unruptured VADA patients may be attributable to thrombotic occlusion of the stenotic lesion, distal emboli, to further occlusion of perforating arteries originating from the VA due to progression of the dissection.

The major limitations of our study were the small number of cases and the retrospective nature of the study. 


\section{Endovascular Treatment of Intracranial Vertebral Artery Dissecting Aneurysms}

\section{CONCLUSION}

In conclusion, endovascular treatment of VADA appears to be safe and effective in both ruptured and unruptured cases. Follow-up angiographic study is needed because parent artery recanalization or aneurysmal recurrence can occur.

\section{References}

1. Jin SC, Kwon DH, Choi CG, Ahn JS, Kwun BD. Endo-vascular strategies for vertebrobasilar dissecting aneurysms. AJNR Am J Neuroradiol 2009;30:1518-1523

2. S.S.M.P. Shrestha Prabin, Shibukawa Masaaki, Kiura Yoshihiro, Okazaki Takahito, Sugiyama Kazuhiko, Kurisu Kaoru. Endovascular coil occlusion of ruptured vertebral artery dissecting aneurysm: a case report. Neurology Asia 2009;14:149-152

3. Kai Y, Nishi T, Watanabe M, Morioka M, Hirano T, Yano S, et al. Strategy for treating unruptured vertebral artery dissecting aneurysms. Neurosurgery 2011;69:1085-1091

4. Lv X, Jiang C, Li Y, Wu Z. Clinical outcomes of ruptured and unruptured vertebral artery-posterior inferior cerebellar artery complex dissecting aneurysms after endovascular embolization. AJNR Am J Neuroradiol 2010;31:1232-1235

5. Fukunaga A, Tabuse M, Naritaka H, Nakamura T, Akiyama T. Spontaneous resolution of nontraumatic bilateral intracranial vertebral artery dissections. Neurol Med Chir (Tokyo) 2002;42: 491-495

6. Naito I, Iwai T, Sasaki T. Management of intracranial vertebral artery dissections initially presenting without subarachnoid hemorrhage. Neurosurgery 2002;51:930-938

7. Kitanaka C, Tanaka J, Kuwahara M, Teraoka A, Sasaki T, Takakura K, et al. Nonsurgical treatment of unruptured intracranial vertebral artery dissection with serial follow-up angiography. J Neurosurg 1994;80:667-674

8. Sasayama T, Korosue K. Spontaneous resolution of the unruptured dissecting aneurysms of bilateral vertebral arteries with conservative treatment: analysis of the transient enlargement of aneurysmal dilatation. No Shinkei Geka 2000;28:47-51

9. Coley SC, Clifton A. Dissecting vertebral artery aneurysm: diagnosis and coil embolization. Br J Radiol 1999;72:408-411

10. Ihn YK, Sung JH, Byun JH. Antegrade recanalization of parent artery after internal trapping of ruptured vertebral artery dissecting aneurysm. J Korean Neurosurg Soc 2012;51:301-304
11. Kurata A, Ohmomo T, Miyasaka Y, Fujii K, Kan S, Kitahara T. Coil embolization for the treatment of ruptured dissecting vertebral aneurysms. AJNR Am J Neuroradiol 2001;22: 11-18

12. Iihara K, Sakai N, Murao K, Sakai H, Higashi T, Kogure S, et al. Dissecting aneurysms of the vertebral artery: a management strategy. J Neurosurg 2002;9:259-267

13. Yuki I, Murayama Y, Vinuela F. Endovascular management of dissecting vertebrobasilar artery aneurysms in patients presenting with acute subarachnoid hemorrhage. J Neurosurg 2005;103:649655

14. Rabinov JD, Hellinger FR, Morris PP, Ogilvy CS, Putman CM. Endovascular management of vertebrobasilar dissecting aneurysms. AJNR Am J Neuroradiol 2003;24:1421-1428

15. Hamada J, Kai Y, Morioka M, Yano S, Todaka T, Ushio Y. Multimodal treatment of ruptured dissecting aneurysms of the vertebral artery during the acute stage. J Neurosurg 2003;99:960966

16. Anxionnat R, de Melo Neto JF, Bracard S, Lacour JC, Pinelli C, Civit T, et al. Treatment of hemorrhagic intracranial dissections. Neurosurgery 2003;53:289-301

17. Yamaura I, Tani E, Yokota M, Nakano A, Fukami M, Kaba K, et al. Endovascular treatment of ruptured dissecting aneurysms aimed at occlusion of the dissected site by using Guglielmi detachable coils. J Neurosurg 1999;90:853-856

18. Halbach VV, Higashida RT, Dowd CF, Fraser, KW, Smith TP, Teitelbaum GP, et al. Endovascular treatment of vertebral artery dissections and pseudoaneurysms. J Neurosurg 1993;79: 183-191

19. Baik SK, Kim YS, Lee HJ, Park J, Kang DS. Antegrade recanalization of parent artery in internal trapping of vertebral artery dissecting aneurysm: a case report. Surg Neurol 2007;68: 108-111

20. Sawada M, Kaku Y, Yoshimura S, Kawaguchi M, Matsuhisa T, Hirata T, et al. Antegrade recanalization of a completely embolized vertebral artery after endovascular treatment of a ruptured intracranial dissecting aneurysm. Report of two cases. $J$ Neurosurg 2005;102:161-166

21. Canton G, Levy DI, Lasheras JC, Nelson PK. Flow changes caused by the sequential placement of stents across the neck of sidewall cerebral aneurysms. J Neurosurg 2005;103:891-902

22. Lopes D, Sani S. Histological postmortem study of an internal carotid artery aneurysm treated with the Neuroform stent. Neurosurgery 2005;56:416

23. Yoon WK, Kim YW, Kim SR, Park IS, Kim SD, Jo KW, et al. Angiographic and clinical outcomes of stent-alone treatment for spontaneous vertebrobasilar dissecting aneurysm. Acta Neurochir (Wien) 2010;152:1477-1486 